\title{
Dual Hop Wireless Communications over Nakagami Fading
}

\author{
Dimitris A. Zogas*, George K. Karagiannidis ${ }^{\dagger}$, Nikos C. Sagias ${ }^{\ddagger}$, \\ Theodoros A. Tsiftsis*, P. Takis Mathiopoulos ${ }^{\dagger}$ and Stavros A. Kotsopoulos* \\ *Electrical \& Computer, Engineering Department, University of Patras, Rion, 26442, Patras, Hellas \\ Email: zogas@space.noa.gr, \{tsiftsis,kotsop\}@ee.upatras.gr

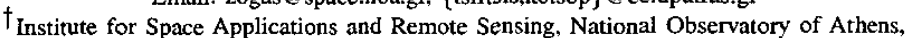 \\ Metaxa \& Vas. Pavlou Street, Palea Penteli, 15236, Athens, Hellas \\ Email: \{gkarag,mathio\}@ space.noa.gr \\ $\ddagger_{\text {Laboratory of Electronics. Physics Department, University of Athens, Panepistimiopolis, 15784, Athens, Hellas }}$ \\ Email: nsagias@space.noa.gr
}

\begin{abstract}
We study the performance of dual-hop wireless communications systems with non-regenerative relays over Nakagami- $m$ fading channels. Deriving tight upper bounds for the end-to-end SNR, important performance criteria such as average end-to-end SNR and error probability are studied. Numerical and computer simulations results are presented to verify the accuracy of the proposed mathematical analysis and to compare our results with previously published ones.
\end{abstract}

\section{INTRODUCTION}

Recently, research efforts have been concentrated on the investigation of multihop wireless communications systems, which seem to provide a potential for broader and more efficient coverage in traditional (bent pipe satellites and microwave links) and modern (ad-hoc, cellular, WLAN and hybrid) wireless networks [1]-[4](and references therein). In such networks, several intermediate terminals operate as relays between the source and the destination. If the relays just amplify and re-transmit the information signal, they are called "non-regenerative", while when the relaying node decodes the signal and then transmits the detected version to the next node, it is called "regenerative" [5]. In this way, the negative effects of the wireless channel impairments are mitigated. Scanning in the up-to-date open technical literature, the number of published works concerning the performance analysis of multihop wireless communications systems is relatively small. Hasna and Alouini, studied the outage and the error performance of dual-hop systems with regenerative and non-regenerative relays over Rayleigh [5], [6] and Nakagami-m [7] fading channels. Recently, in [8], the same authors presented a useful and general analytical framework for the evaluation of the endto-end outage probability of multihop wireless systems with non-regenerative relays over Nakagami- $m$ fading channels. In [5]-[8], the analysis is based on an upper bound for the end-toend signal-to-noise ratio (SNR) of the wireless system, which leads to lower bounds on the outage and the error performance.

In this paper, the performance of dual-hop wireless communications systems with non-regenerative relays, operating over independent but not necessarily identically distributed (i.d.) Nakagami- $m$ fading channels is studied. Using a well-known inequality between the arithmetic, geometric and harmonic mean of positive random variables (rv), we present tight upper bounds for the end-to-end SNR, which can be efficiently used to evaluate in closed-forms the moments of the end-to-end SNR and the average error probability at low SNR. Numerical and computer simulations results depict the accuracy of the mathematical analysis, as well as the improvement of the proposed approach, compared to previously published formulas.

The remainder of this paper is organized as follows. The next section presents the system and channel model under consideration. In Sections III and IV, the statistics of the endto-end SNR and the error performance of the system are studied, respectively. Finally, some concluding remarks are offered in Section V.

\section{System AND Channel Model}

A dual-hop wireless communications system with a nonregenerative relay is considered, operating over independent but not necessarily i.d. Nakagami- $m$ fading channels. Assume that terminal $T_{1}$ transmits a signal with an average power normalized to unity, the received signal at terminal $T_{2}$ can be written as

$$
r_{T_{2}}=a_{1} s+n_{1}
$$

where $a_{1}$ is the fading amplitude of the channel between terminals $T_{1}$ and $T_{2}$, modeled as a Nakagami-m rv and $n_{1}$ is the additive white Gaussian noise (AWGN) with singlesided power spectral density (psd) $N_{0}$. The signal $r_{T_{2}}$ is then multiplied by the gain $g$ of terminal $T_{2}$ and re-transmitted to terminal $T_{3}$, where the received signal can be written as

$$
r_{T_{3}}=g a_{2}\left(a_{1} s+n_{1}\right)+n_{2}
$$

where $a_{2}$ is the fading amplitude of the channel between $T_{2}$ and $T_{3}$, and $n_{2}$ is the AWGN with single-sided psd $N_{0}$. Setting the gain to [9]

$$
g^{2}=\frac{1}{a_{1}^{2}+N_{0}}
$$

with aim to invert the fading effect of the first channel, while limiting the output power of the relay, if the fading amplitude 
of the first hop is low, the overall SNR at the receiving end can be written as

$$
\gamma_{\text {end }}=\frac{\frac{a_{1}^{2}}{N_{0}} \frac{a_{2}^{2}}{N_{0}}}{\frac{a_{2}^{2}}{N_{0}}+\frac{1}{g^{2} N_{0}}}=\frac{\gamma_{1} \gamma_{2}}{\gamma_{1}+\gamma_{2}+1}
$$

where $\gamma_{i}=a_{i}^{2} / N_{0}, i=1,2$, is the instantaneous SNR of the $i$ th hop. Equation (4) can be rewritten as

$$
\frac{1}{\gamma_{e n d}}=\frac{1}{\gamma_{1}}+\frac{1}{\gamma_{2}}+\frac{1}{\gamma_{1} \gamma_{2}} \text {. }
$$

While the fading envelope $a_{i}$ is a Nakagami- $m \mathrm{rv}, \gamma_{i}$ is a gamma distributed $\mathrm{rv}$, with probability density function (pdf) given by [10, eq. (2.21)]

$$
f_{\gamma_{i}}\left(\gamma_{i}\right)=\frac{m_{i}^{m_{i}}}{\bar{\gamma}_{i}^{m_{i}} \Gamma\left(m_{i}\right)} \gamma_{i}^{m_{i}-1} \exp \left[-\frac{m_{i} \gamma_{i}}{\bar{\gamma}_{i}}\right]
$$

where $\Gamma(\cdot)$ is the Gamma function [11, eq. $(8.310 .1)], m_{i}$ is the Nakagami- $m$ parameter describing the fading severity of the $i$ th hop and $\bar{\gamma}_{i}$ is the average SNR of the $i$ th hop defined as $\bar{\gamma}_{i}=\overline{a_{i}^{2}} / N_{0}$.

\section{STATISTICS OF THE END-TO-END SNR}

In order to study the average end-to-end SNR of the dual-hop wireless system of interest, we bound the overall SNR, $\gamma_{e n d}$, using a well-known inequality between arithmetic, geometric and harmonic mean of $N$ positive rvs.

Definition: The arithmetic, geometric and harmonic mean of $N$ positive rvs, $\left\{x_{n}\right\}_{n \in \mathcal{Z}_{N}}$, is

$$
\begin{gathered}
\mathcal{A}(N) \triangleq \frac{1}{N} \sum_{k \in \mathcal{Z}_{N}} x_{k}, \\
\mathcal{G}(N) \triangleq\left(\prod_{k \in \mathcal{Z}_{N}} x_{k}\right)^{\frac{1}{N}}
\end{gathered}
$$

and

$$
\mathcal{H}(N) \triangleq\left(\frac{1}{N} \sum_{k \in Z_{N}} \frac{1}{x_{k}}\right)^{-1}
$$

respectively.

Theorem 1: The arithmetic, geometric and harmonic mean of $N$ positive rvs, $\left\{x_{n}\right\}_{n \in \mathcal{Z}_{N}}$, satisfy the inequality

\section{Proof: See [12].}

$$
\mathcal{A}(N) \geq \mathcal{G}(N) \geq \mathcal{H}(N) \text {. }
$$

Applying (7) $\left(\gamma_{1}+\gamma_{2} \geq 2 \sqrt{\gamma_{1} \gamma_{2}}\right)$ to (4), results to an upper bound for the overall SNR given by

$$
\gamma_{e n d} \leq \gamma_{e n d 1}=\frac{\gamma_{1} \gamma_{2}}{2 \sqrt{\gamma_{1} \gamma_{2}}+1} .
$$

The form of $\gamma_{e n d 1}$ has the advantage of mathematical tractability over that in (4) in addition to being a tight upper bound for $\gamma_{e n d}$. By definition, the $n$th moment of $\gamma_{e n d 1}$ is

$$
\begin{aligned}
E\left\langle\gamma_{\text {end } 1}^{n}\right\rangle=\int_{0}^{\infty} \int_{0}^{\infty} & \left(\frac{\gamma_{1} \gamma_{2}}{2 \sqrt{\gamma_{1} \gamma_{2}}+1}\right)^{n} \\
& \times f_{\gamma_{1}}\left(\gamma_{1}\right) f_{\gamma_{2}}\left(\gamma_{2}\right) d \gamma_{1} d \gamma_{2}
\end{aligned}
$$

where $E\langle\cdot\rangle$ denotes expectation. Equation (9) can be written in closed-form as (see Appendix I)

$$
\begin{aligned}
& E\left\langle\gamma_{e n d 1}^{n}\right\rangle= \frac{2^{n-1}}{\pi \Gamma(n) \Gamma\left(m_{1}\right) \Gamma\left(m_{2}\right)}\left(\frac{\bar{\gamma}_{1} \bar{\gamma}_{2}}{m_{1} m_{2}}\right)^{n} \\
& \times G_{4,2}^{2,4}\left(\left.4 \frac{\bar{\gamma}_{1} \bar{\gamma}_{2}}{m_{1} m_{2}}\right|^{\frac{1-n}{2}, \frac{2-n}{2}, 1-m_{1}-n, 1-m_{2}-n}\right) \\
& 0, \frac{1}{2}
\end{aligned}
$$

where $G_{p, q}^{m, n}\left(x \mid \begin{array}{l}a_{1}, \ldots, a_{p} \\ b_{1}, \ldots, b_{q}\end{array}\right)$ is the Meijer's G-function [11, ch. 9.3]. Setting $n=1$ to (10), the average end-to-end SNR $\bar{\gamma}_{\text {end } 1}$ is derived as

$$
\begin{aligned}
\bar{\gamma}_{e n d 1}= & \frac{\bar{\gamma}_{1} \bar{\gamma}_{2}}{\pi \Gamma\left(m_{1}\right) \Gamma\left(m_{2}\right) m_{1} m_{2}} \\
& \times \mathrm{G}_{4,2}^{2,4}\left(4 \frac{\bar{\gamma}_{1} \bar{\gamma}_{2}}{m_{1} m_{2}} \mid \begin{array}{c}
0,0,-m_{1},-m_{2} \\
0, \frac{1}{2}
\end{array}\right) .
\end{aligned}
$$

If the two links are identical, (i.e., $\bar{\gamma}_{1}=\bar{\gamma}_{2}=\bar{\gamma}$ and $\left.m_{1}=m_{2}=m\right)$, (11) reduces to

$$
\begin{aligned}
\bar{\gamma}_{e n d 1}= & \frac{1}{\pi}\left[\frac{\bar{\gamma}}{\Gamma(m) m}\right]^{2} \\
& \times G_{4,2}^{2,4}\left(\left(2 \frac{\bar{\gamma}}{m}\right)^{2} \begin{array}{c}
0,0,-m,-m \\
0, \frac{1}{2}
\end{array}\right)
\end{aligned}
$$

and for the Rayleigh fading case (12) is written as

$$
\bar{\gamma}_{e n d 1}=\frac{\bar{\gamma}^{2}}{\pi} \mathrm{G}_{4,2}^{2,4}\left(4 \bar{\gamma}^{2} \mid \begin{array}{c}
0,0,-1,-1 \\
0, \frac{1}{2}
\end{array}\right) \text {. }
$$

It must be noted here, that (10) can be used to study several other quality measures, such as the kurtosis and the skewness, that characterize the distribution of $\gamma_{e n d 1}$ and consequently the distribution of $\gamma_{e n d}$. Kurtosis is a measure of the peakedness of a distribution, namely, the higher the kurtosis the lower the concentration around its mode [13]. For high values of kurtosis the pdf is called leptokurtic and for low values platykurtic. Leptokurtic rvs have typically a spiky pdf with heavy tail. Skewness measures the deviation of the distribution from symmetry. If the distribution has a longer tail less than the maximum, the function has negative skewness. Otherwise, the skewness is positive. The kurtosis and the skewness are given by [14]

$$
\kappa=\frac{E\left\langle\left(\gamma_{e n d 1}-\bar{\gamma}_{e n d 1}\right)^{4}\right\rangle}{\left[E\left\langle\left(\gamma_{e n d 1}-\bar{\gamma}_{e n d 1}\right)^{2}\right\rangle\right]^{2}}-3
$$

and

$$
s k=\frac{E\left\langle\left(\gamma_{e n d 1}-\bar{\gamma}_{e n d 1}\right)^{3}\right\rangle}{\left[E\left\langle\left(\gamma_{e n d 1}-\bar{\gamma}_{e n d 1}\right)^{2}\right\rangle\right]^{3 / 2}},
$$

respectively. The term $E\left\langle\left(\gamma_{e n d 1}-\bar{\gamma}_{e n d 1}\right)^{k}\right\rangle$ is the $k$-central moment of $\gamma_{\text {end } 1}$ and can be obtained as

$E\left\langle\left(\gamma_{e n d 1}-\bar{\gamma}_{e n d 1}\right)^{k}\right\rangle=\sum_{i=0}^{k}\left(\begin{array}{c}k \\ i\end{array}\right)(-1)^{k-i} \bar{\gamma}_{e n d 1}^{k-i} E\left\langle\gamma_{e n d 1}^{i}\right\rangle$. 


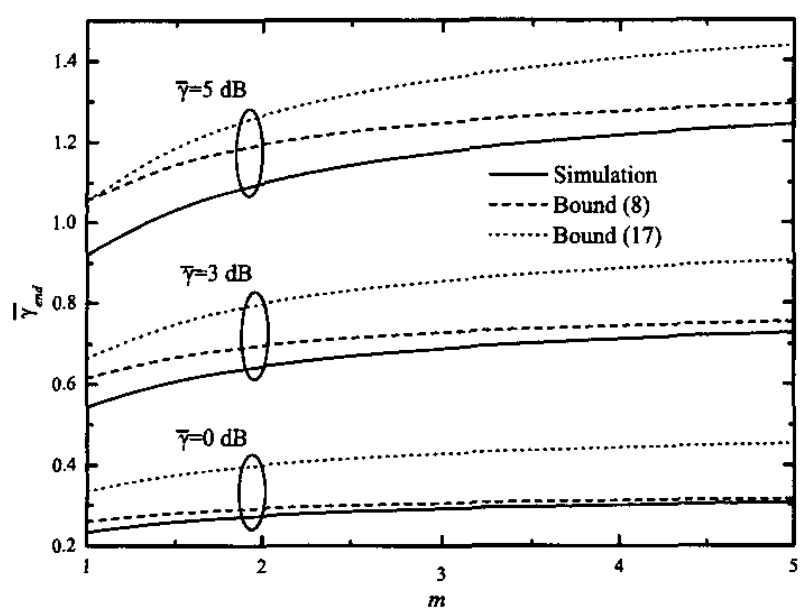

Fig. 1. Comparison of the average overall SNR versus the fading parameter $m$, for end-to-end SNR's given by (17) and (8).

Using (12), Fig. 1 plots the average overall SNR versus the fading parameter $m$ for several values of $\bar{\gamma}$. For comparison purposes, Fig. 1 also plots the average end-to-end SNR for the exact end-to-end SNR given by (4) and for the upper bound proposed in [7], i.e.,

$$
\gamma_{e q}=\frac{\gamma_{1} \gamma_{2}}{\gamma_{1}+\gamma_{2}}
$$

both obtained via Monte Carlo simulations. The bound proposed in this paper gets more tight as $m$ increases and $\bar{\gamma}$ decreases, while variations of $m$ and as $\bar{\gamma}$ have minor effect on the average overall SNR performance of (17).

\section{AVERAGE Symbol ERROR PROBABILITy (ASEP) AT LOW SNR}

In this section, deriving an efficient upper bound for the endto-end SNR, we present an approach for the error performance of dual-hop systems especially at low SNR. The basic idea is that it is always best to reduce the transmitted power to the minimum level for the receiver's operation within its acceptable range. Reducing the transmitted power not only extends the battery life of the wireless device, but also increases the capacity of the wireless network because the same channel can be reused more often to reduce the interference [1]. Moreover, it has been observed that in the forthcoming generations of mobile wireless systems, almost $40 \%$ of the users will experience receiver SNR levels below $0 \mathrm{~dB}$ while less than $10 \%$ display levels above $10 \mathrm{~dB}$ [15]. Based on this observation and given the importance of energy-consumption and low power-transmission, we present an approach for the ASEP analysis at low SNR.

Given the harmonic mean $\mathcal{H}(3)$ of the three quantities $\gamma_{1}$, $\gamma_{2}$ and $\gamma_{1} \gamma_{2}$, (5) can be rewritten as

$$
\frac{1}{\gamma_{\text {end }}}=\frac{3}{\mathcal{H}(3)} \text {. }
$$

Using (7), the end-to-end SNR of the dual-hop system can be upper bounded as

$$
\gamma_{\text {end }} \leq \gamma_{\text {end } 2}=\frac{\mathcal{G}(3)}{3}=\frac{1}{3}\left(\gamma_{1} \gamma_{2}\right)^{2 / 3}
$$

The form of $\gamma_{e n d 2}$ has also the advantage of mathematical tractability over that in (4), in addition to be a upper bound for $\gamma_{\text {end }}$. This will lead to a tight lower bound for the ASEP, especially at low SNRs.

The moment generating function (mgf) of $\gamma_{e n d 2}$, $\mathcal{M}_{\text {end2 } 2}(s) \triangleq E\left\langle\exp \left(-s \gamma_{e n d 2}\right)\right\rangle$ is evaluated in closedform solving the double integral

$$
\begin{aligned}
\mathcal{M}_{\gamma_{\text {end } 2}}(s)=\int_{0}^{\infty} \int_{0}^{\infty} & \exp \left(-\frac{s}{3} \gamma_{1}^{2 / 3} \gamma_{2}^{2 / 3}\right) \\
& \times f_{\gamma_{1}}\left(\gamma_{1}\right) f_{\gamma_{2}}\left(\gamma_{2}\right) d \gamma_{1} d \gamma_{2}
\end{aligned}
$$

as (see Appendix II)

$$
\begin{aligned}
& \mathcal{M}_{\gamma_{\text {end } 2}}(s)=\frac{\sqrt{3} 2^{m_{1}+m_{2}-3}}{\pi^{2} \Gamma\left(m_{1}\right) \Gamma\left(m_{2}\right)} \\
& \quad \times \mathrm{G}_{4,3}^{3,4}\left(\frac{2^{4} s^{3}}{3^{6}}\left(\frac{\bar{\gamma}_{1} \bar{\gamma}_{2}}{m_{1} m_{2}}\right)^{2} \mid \begin{array}{c}
\frac{1-m_{1}}{2}, \frac{2-m_{1}}{2}, \frac{1-m_{2}}{2}, \frac{2-m_{2}}{2}, \\
0, \frac{1}{3}, \frac{2}{3}
\end{array}\right) .
\end{aligned}
$$

For identical links (21) is written as

$$
\begin{aligned}
& \mathcal{M}_{\gamma_{\text {end } 2}}(s)=\frac{\sqrt{3} 2^{2 m-3}}{\pi^{2} \Gamma^{2}(m)} \\
& \times G_{4,3}^{3,4}\left(\frac{2^{4} s^{3} \vec{\gamma}^{4}}{3^{6} m^{4}} \mid \begin{array}{c}
\frac{1-m}{2}, \frac{2-m}{2}, \frac{1-m}{2}, \frac{2-m}{2}, \\
0, \frac{3}{3}, \frac{2}{3}
\end{array}\right)
\end{aligned}
$$

and for the Rayleigh case (22) reduces to

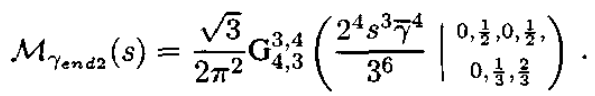

Having the mgf of $\gamma_{\text {end } 2}$ in closed-form and using the mgfbased approach for the error analysis of digital communications systems over fading channels $[10$, ch. 1], the ASEP can be evaluated directly for non-coherent and differential binary signaling (e.g. NFSK and DPSK), since for all other cases, (e.g. $M$-AM, $M$-PSK, $M$-QAM, BFSK and $M$-DPSK), single integrals with finite limits have to be evaluated via numerical integration. For example, the ASEP for DPSK is given by $\bar{P}_{s e}=0.5 \mathcal{M}_{\gamma_{\text {end }}}(1)$.

Using (22), Figs. 2 and 3 depict the error performance of binary DPSK and PSK, respectively, for the dual-hop system of interest at low SNR. Curves for the exact error performance, obtained via Monte Carlo simulations, and for the error performance assuming end-to-end SNR given by (17), obtained using [7, eq. (29)], are also depicted for comparison reasons. It is evident that the bound proposed in this paper is more efficient at low SNR comparing to (17). 


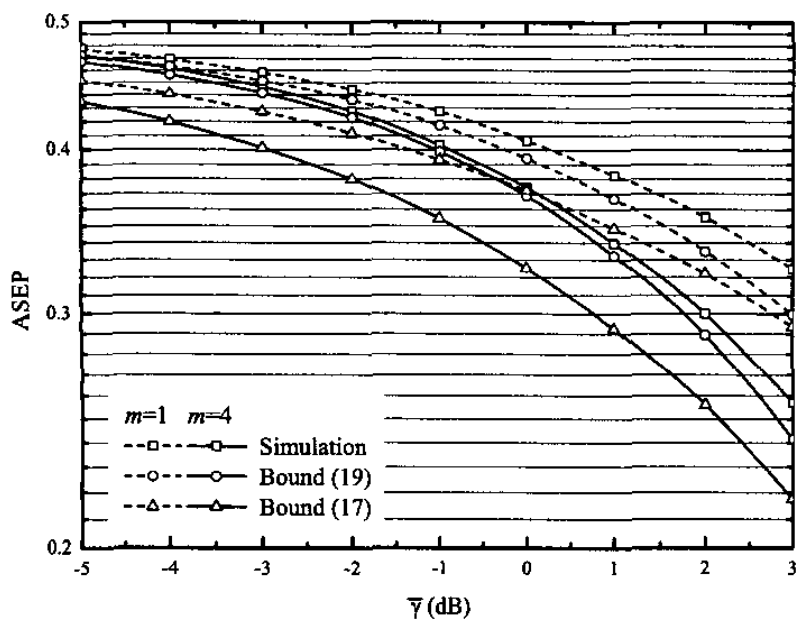

Fig. 2. Comparison of the error performance of binary DPSK, for end-to-end SNR's given by (17) and (19).

\section{CONCLUSIONS}

In this paper, the performance of dual-hop wireless communications systems with non-regenerative relays and operating over Nakagami- $m$ fading channels was studied. More specifically, the average end-to-end SNR and error probability were studied deriving upper bounds for the end-to-end SNR of the system. Numerical and simulations results showed the accuracy of the proposed mathematical analysis, as well as the improvement achieved using the proposed approach, compared to previously published results.

\section{APPENDIX I}

\section{DERIVATION OF (10)}

Substituting (6) into (9), the $n$th moment of $\gamma_{e n d 1}$ can be written as

$$
\begin{aligned}
E\left\langle\gamma_{\text {end } 1}^{n}\right\rangle & =\frac{1}{\Gamma\left(m_{1}\right) \Gamma\left(m_{2}\right)}\left(\frac{m_{1}}{\bar{\gamma}_{1}}\right)^{m_{1}}\left(\frac{m_{2}}{\bar{\gamma}_{2}}\right)^{m_{2}} \\
& \times \int_{0}^{\infty} \gamma_{2}^{n+m_{2}-1} \exp \left(-\frac{m_{2}}{\bar{\gamma}_{2}} \gamma_{2}\right) \\
& \times \int_{0}^{\infty} \frac{\gamma_{1}^{n+m_{1}-1}}{\left(2 \sqrt{\gamma_{1} \gamma_{2}}+1\right)^{n}} \exp \left(-\frac{m_{1}}{\bar{\gamma}_{1}} \gamma_{1}\right) d \gamma_{1} d \gamma_{2}
\end{aligned}
$$

The integrand in the first integration in (I.1), i.e., the one on $\gamma_{1}$, using [16, eq. (10)], [16, eq. (11)] can be written in terms of the Meijer's G-function as

$$
\begin{aligned}
I_{1}^{I}=\frac{1}{\Gamma(n)} \int_{0}^{\infty} & \gamma_{1}^{n+m_{1}-1} \mathrm{G}_{0,1}^{1,0}\left(\frac{m_{1}}{\bar{\gamma}_{1}} \gamma_{1} \mid \begin{array}{l}
- \\
0
\end{array}\right) \\
& \times \mathbf{G}_{1,1}^{1,1}\left(\left.2 \sqrt{\gamma_{2} \gamma_{1}}\right|^{-n+1}\right) d \gamma_{1} .
\end{aligned}
$$

Integrals of the form of (I.2) can evaluated in closed-form using $[16$, eq. (21)] as shown at the top of the next page

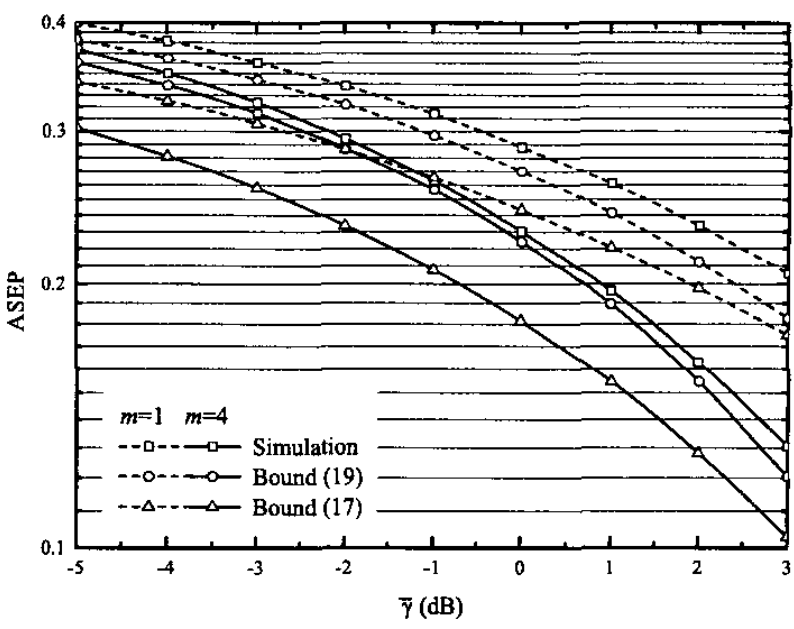

Fig. 3. Comparison of the error performance of binary PSK, for end-to-end SNR's given by (17) and (19).

where

$$
\begin{array}{ll}
c^{*}=m+n-\frac{p+q}{2}, & \mu=\sum_{j=1}^{q} b_{j}-\sum_{j=1}^{p} a_{j}+\frac{p-q}{2}+1, \\
b^{*}=s+t-\frac{u+v}{2}, & \varrho=\sum_{j=1}^{\nu} d_{j}-\sum_{j=1}^{u} c_{j}+\frac{u-v}{2}+1
\end{array}
$$

and

$$
\Delta(k, h)=\frac{h}{k}, \frac{h+1}{k}, \ldots, \frac{h+k-1}{k} .
$$

Using (I.3), the integral in (I.2) can be solved as

$$
\begin{aligned}
& I_{1}^{I}= \frac{2^{n-1}}{\pi \Gamma(n)}\left(\frac{\bar{\gamma}_{1}}{m_{1}}\right)^{m_{1}+n} \\
& \times \mathrm{G}_{3,2}^{2,3}\left(4 \frac{\bar{\gamma}_{1}}{m_{1}} \gamma_{2}\right. \\
&\left.\begin{array}{c}
\frac{1-n}{2}, \frac{2-n}{2}, 1-m_{1}-n \\
0, \frac{1}{2}
\end{array}\right) .
\end{aligned}
$$

The second integral in (I.1) can now be written as

$$
\begin{aligned}
& I_{2}^{I}= \int_{0}^{\infty} \gamma_{2}^{m_{2}-1} \mathrm{G}_{0,1}^{1,0}\left(\frac{m_{2}}{\bar{\gamma}_{2}} \gamma_{2} \mid \begin{array}{l}
- \\
0
\end{array}\right) \\
& \times \mathrm{G}_{3,2}^{2,3}\left(4 \frac{\bar{\gamma}_{1}}{m_{1}} \gamma_{2}\right. \\
&\left.\begin{array}{c}
\frac{1-n}{2}, \frac{2-n}{2}, 1-m_{1}-n \\
0, \frac{1}{2}
\end{array}\right) d \gamma_{2} .
\end{aligned}
$$

and using (I.3) yields

$$
\left.\begin{array}{rl}
I_{2}^{I} & =\left(\frac{\bar{\gamma}_{2}}{m_{2}}\right)^{m_{2}+n} \\
& \times \mathrm{G}_{4,2}^{2,4}\left(\left.4 \frac{\bar{\gamma}_{1} \bar{\gamma}_{2}}{m_{1} m_{2}}\right|^{\frac{1-n}{2}, \frac{2-n}{2}, 1-m_{1}-n, 1-m_{2}-n}\right) . \\
0, \frac{1}{2}
\end{array}\right) .
$$

Finally, combining (I.1), (I.4) and (I.6) leads to the closedform expression for $E\left\langle\gamma_{e n d 1}^{n}\right\rangle$ given by (10). 


$$
\begin{aligned}
& \int_{0}^{\infty} x^{\eta-1} \mathrm{G}_{u, v}^{s, t}\left[\begin{array}{l|l}
\sigma x & \left.\begin{array}{l}
\left\{c_{u}\right\} \\
\left\{d_{v}\right\}
\end{array}\right] \mathrm{G}_{p, q}^{m, n}\left[\omega x^{\frac{l}{k}} \mid \begin{array}{l}
\left\{a_{p}\right\} \\
\left\{b_{q}\right\}
\end{array}\right] d x=\frac{k^{\mu} l^{\varrho+\eta(v-u)-1} \sigma^{-\eta}}{(2 \pi)^{b^{*}(l-1)+c^{*}(k-1)}} \\
\times \mathrm{G}_{k p+l v, k q+l u}^{k m+l t, k n+l s}\left[\frac{\omega^{k} k^{k(p-q)}}{\sigma^{l} l^{l(u-v)}} \mid \begin{array}{l}
\Delta\left(k, a_{1}\right), \ldots, \Delta\left(k, a_{n}\right), \Delta\left(l, 1-\eta-d_{1}\right), \ldots, \Delta\left(l, 1-\eta-d_{\nu}\right), \Delta\left(k, a_{n+1}\right), \ldots, \Delta\left(k, a_{p}\right) \\
\Delta\left(k, b_{1}\right), \ldots, \Delta\left(k, b_{m}\right), \Delta\left(l, 1-\eta-c_{1}\right), \ldots, \Delta\left(l, 1-\eta-c_{u}\right), \Delta\left(k, b_{m+1}\right), \ldots, \Delta\left(k, b_{q}\right)
\end{array}\right]
\end{array}\right.
\end{aligned}
$$

\section{APPENDIX II \\ DERIVATION OF (21)}

The mgf of $\gamma_{e n d 2}$ is given by

$$
\begin{aligned}
\mathcal{M}_{\gamma_{\text {end }}}(s)=\int_{0}^{\infty} \int_{0}^{\infty} & \exp \left(-\frac{s}{3} \gamma_{1}^{2 / 3} \gamma_{2}^{2 / 3}\right) \\
& \times f_{\gamma_{1}}\left(\gamma_{1}\right) f_{\gamma_{2}}\left(\gamma_{2}\right) d \gamma_{1} d \gamma_{2}
\end{aligned}
$$

The first integration in (II.1), i.e., the one on $\gamma_{1}$, is of the form

$$
\begin{aligned}
& I_{1}{ }^{I I}=\frac{1}{\Gamma\left(m_{1}\right)}\left(\frac{m_{1}}{\bar{\gamma}_{1}}\right)^{m_{1}} \\
& \quad \times \int_{0}^{\infty} \gamma_{1}^{m_{1}-1} \exp \left(-\frac{m_{1}}{\bar{\gamma}_{1}} \gamma_{1}\right) \exp \left(-\frac{s}{3} \gamma_{1}^{2 / 3} \gamma_{2}^{2 / 3}\right) d \gamma_{1}
\end{aligned}
$$

which can be written in terms of the Meijer's G-function as

$$
\begin{aligned}
I_{1}^{I I}= & \frac{1}{\Gamma\left(m_{1}\right)}\left(\frac{m_{1}}{\bar{\gamma}_{1}}\right)^{m_{1}} \\
& \times \int_{0}^{\infty} \gamma_{1}^{m_{1}-1} \mathrm{G}_{0,1}^{1,0}\left(\frac{m_{1}}{\bar{\gamma}_{1}} \gamma_{1}{ }_{0}^{-}\right) \\
& \quad \times \mathrm{G}_{0,1}^{1,0}\left(\begin{array}{ll|l}
\frac{s \gamma_{2}^{2 / 3}}{3} \gamma_{1}^{2 / 3} & - \\
&
\end{array} \quad d \gamma_{1} .\right.
\end{aligned}
$$

This integral can be solved using the formula in (I.3) as

$$
\begin{aligned}
I_{1}^{I I}= & \frac{\sqrt{3} 2^{m_{1}-1 / 2}}{(2 \pi)^{3 / 2} \Gamma\left(m_{1}\right)} \\
& \times \mathrm{G}_{2,3}^{3,2}\left(\frac{4 s^{3}}{3^{6}}\left(\frac{\bar{\gamma}_{1}}{m_{1}}\right)^{2} \gamma_{2} \mid \begin{array}{c}
\frac{1-m_{1}}{2}, \frac{2-m_{1}}{2} \\
0, \frac{2}{3}, \frac{2}{3}
\end{array}\right) .
\end{aligned}
$$

Equation (II.1) can now be written as

$$
\begin{aligned}
& \mathcal{M}_{\gamma_{\text {end }}}(s)=\frac{\sqrt{3} 2^{m_{1}-1 / 2}}{(2 \pi)^{3 / 2} \Gamma\left(m_{1}\right) \Gamma\left(m_{2}\right)}\left(\frac{m_{2}}{\overline{\bar{\gamma}}_{2}}\right)^{m_{2}} \\
& \times \int_{0}^{\infty} \gamma_{2}^{m_{2}-1} \mathrm{G}_{0,1}^{1,0}\left(\frac{m_{2}}{\bar{\gamma}_{2}} \gamma_{2} \mid \begin{array}{c}
- \\
0
\end{array}\right) \\
& \times \mathrm{G}_{2,3}^{3,2}\left(\begin{array}{l|l}
\frac{4 s^{3}}{3^{6}}\left(\frac{\bar{\gamma}_{1}}{m_{1}}\right)^{2} \gamma_{2} & \begin{array}{c}
\frac{1-m_{1}}{2}, \frac{2-m_{2}}{2} \\
0, \frac{1}{3}, \frac{2}{3}
\end{array}
\end{array}\right) d \gamma_{2} .
\end{aligned}
$$

Using (I.3), the mgf of $\gamma_{e n d 2}$ can be finally written as in (21).

\section{REFERENCES}

[1] Special Issue on "Energy-aware ad hoc wireless networks," IEEE Wirel. Commun, vol. 9, Aug. 2002.

[2] Y.-D. Lin and Y.-C. Hsu, "Multihop cellular: a new architecture for wireless communications." in Proc. IEEE INFOCOM, 2000, Tel-Aviv, Israel, pp. 1273-1282.

[3] V. Emamian and M. Kaveh, "Combating shadowing effects for systems with transmitter diversity by using collaboration among mobile users." in Proc. Intemational Symposium on Communications, Nov. 2001, Taiwan, pp. 105.1-105.4.

[4] H. Yanikomeroglu, "Fixed and mobile relaying technologies for cellular networks," in Proc. 2nd Workshop on Applications and Services in Wireless Networks, July 2002, Paris, France, pp. 75-81.

[5] M. O. Hasna and M.-S. Alouini, "End-to-end performance of transmission systems with relays over Rayleigh fading channels," IEEE Trans. Wirel. Commun., vol. 2, pp. 1126-1131, Nov. 2003.

[6] M. O. Hasna and M.-S. Alouini, "Performance analysis of two-hop relayed transmissions over Rayleigh fading channels," in Proc. IEEE Vehicular Technology Conference, Sep. 2002, pp. 1992-1996.

[7] M. O. Hasna and M.-S. Alouini, "Application of the harmonic mean statistics to the end-to-end performance of transmission systems with relays," in Proc. IEEE Global Telecommunications Conference, Nov. 2002, pp. 1310-1314.

[8] M. O. Hasna and M.-S. Alouini, "Outage probability of multihop transmission over Nakagami fading channels," IEEE Commun. Lett., vol. 7. pp. 216-218, May 2003.

[9] J. N. Laneman and G. W. Wornell, "Energy efficient antenna sharing and relaying for wireless networks," in Proc. IEEE Wireless Computers and Networks Conference, Oct. 2000, pp. 7-12.

[10] M. K. Simon and M.-S. Alouini, Digital Communication over Fading Channels. 1st ed., New York: John Wiley, 2000.

[11] I. S. Gradshteyn and I. M. Ryzhik, Table of Integrals, Series, and Products. 5th ed., New York: Academic, 1994.

[12] A. Stuart and K. Ord, Kendall's Advanced Theory of Statistics Vol. I: Distribution Theory. 6th ed., Edward Amold, 1994.

[13] S. Shamai and S. Verdu, "The impact of frequency-flat fading on the spectral efficiency of CDMA." IEEE Trans. Inform. Theory, vol. 47, pp. 1302-1327, May 2001.

[14] D. Zwillinger and S. Kokoska, Standard Probability and Statistics Tables and Formulae. 2nd ed., CRC Press, 1999.

[15] A. Lozano, A. M. Tulino, and S. Verdu, "Multiple-antenna capacity in the low-power regime," IEEE Trans. Inform. Theory, vol. 49, pp. 25272544, Oct. 2003.

[16] V. S. Adamchik and O. I. Marichev, 'The algorithm for calculating integrals of hypergeometric type functions and its realization to REDUCE system," in Proc. International Conference on Symbolic and Algebraic Computation, Tokyo, Japan, 1990, pp. 212-224. 